\title{
Travel-associated Legionnaires' disease in Europe, 2010
}

B de Jong (birgitta.dejong@ecdc.europa.eu) ${ }^{1}$, L Payne Hallström ${ }^{1}$, E Robesyn ${ }^{1}$, D Ursut ${ }^{1}$, P Zucs ${ }^{1}$, on behalf of ELDSNet (European Legionnaires' Disease Surveillance Network)

1. Surveillance and Response Support Unit, European Centre for Disease Prevention and Control (ECDC), Stockholm, Sweden

Citation style for this article:

de Jong B, Payne Hallström L, Robesyn E, Ursut D, Zucs P, on behalf of ELDSNet (European Legionnaires' Disease Surveillance Network). Travel-associated Legionnaires' disease in Europe, 2010. Euro Surveill. 2013;18(23):pii=20498. Available online: http://www.eurosurveillance.org/ViewArticle.aspx?Articleld=20498

In 2010, the European surveillance network for travelassociated Legionnaires' disease (ELDSNet, previously EWGLINET) received reports of 864 cases of travelassociated Legionnaires' disease, of whom 24 were reported to have had a fatal outcome. As in previous years, a very low proportion of clinical isolates were obtained ( 45 cases, 5.6\%). In the 2010 dataset, male cases outnumbered female cases by $2.6: 1$ and had a median age of 61 years (range: 21-96), while the median age for women was 63 years (range: 12-95). The network identified 100 new clusters in 2010, of which 44 involved only one case from each reporting country and would probably not have been detected by national surveillance schemes alone. The largest cluster (having 14 cases) was associated with a cruise ship. Legionella species were detected at 61 of the 100 accommodation site clusters investigated. The names of five accommodation sites were published on the ECDC website.

\section{Introduction}

Legionnaires' disease is an uncommon form of pneumonia caused by Legionella bacteria. It has no particular clinical features that clearly distinguish it from other types of pneumonia, and laboratory investigations must be carried out to confirm the diagnosis. It normally takes between 2 and 10 days to develop symptoms (typically five to six days) but very rarely, some cases may take two to three weeks to develop symptoms. Patients usually start with a dry cough, fever, headache and sometimes diarrhoea and many go on to get pneumonia. People over the age of 50 years are more at risk than younger people, and males are more at risk than females. Effective antibiotic treatment is available if the diagnosis is made early in the illness. Death due to the disease occurs in about $5 \%$ to $15 \%$ of travellers who develop the disease, depending on their age and health status. Smokers are more at risk than non-smokers [1].

In April 2010, ELDSNet (European Legionnaires' Disease Surveillance Network) was established, when the European surveillance scheme for travel-associated Legionnaires' disease (EWGLINET) was transferred to the European Centre for Disease Prevention

\section{Box}

European Union case definition of Legionnaires' disease

Individual cases of travel-associated Legionnaire

Clinical criteria:

Any person with pneumonia.

Laboratory criteria for case confirmation:

At least one of the following three:

- Isolation of Legionella spp. from respiratory secretions or any normally sterile site;

- Detection of Legionella pneumophila antigen in urine;

- Legionella pneumophila serogroup 1 specific antibody response.

Laboratory criteria for a probable case:

At least one of the following four:

- Detection of Legionella pneumophila antigen in respiratory secretions or lung tissue, e.g. by DFA staining using monoclonal-antibody derived reagents;

- Detection of Legionella spp. nucleic acid in a clinical specimen;

- Legionella pneumophila non-serogroup 1 or other Legionella spp. specific antibody response;

- L. pneumophila serogroup 1 , other serogroups or other Legionella species: single high titre in specific serum antibody.

Epidemiological criteria:

At least one of the following two epidemiological links:

- Environmental exposure;

- Exposure to the same common source.

Case classification

Possible case

NA

\section{Probable case}

Any person meeting the clinical criteria AND at least one positive laboratory test for a probable case OR an epidemiological link.

Confirmed case

Any person meeting the clinical and the laboratory criteria for case confirmation.

DFA: direct fluorescent antibody; NA: not applicable. Source:[2]. 


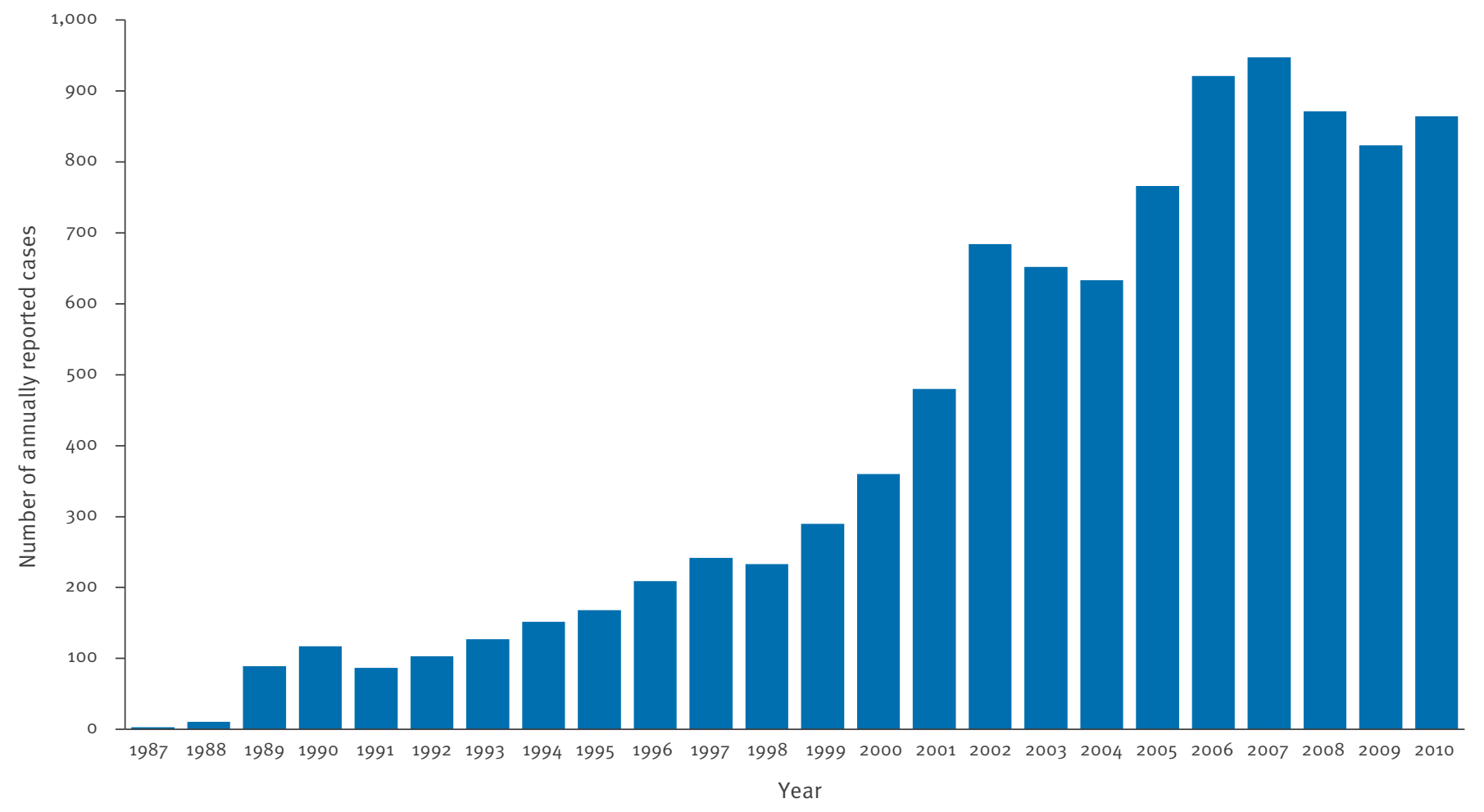

EEA: European Economic Area; EU: European Union. Source: [5].

and Control (ECDC) from the former coordinating centre in London. EWGLINET was established in 1987 by the European Working Group for Legionella Infections, making ELDSNet/EWGLINET the oldest European infectious disease surveillance network. The added value of European surveillance has been clearly demonstrated since the late $80 \mathrm{~s}$ [2-5]. The objectives of this article are to communicate the results of the surveillance of travel-associated Legionnaires' disease in European Union (EU)/European Economic Area (EEA) Member States for cases with onset of disease in 2010.

\section{Methods}

Legionnaires' disease is a statutorily notifiable disease in all EU/EEA Member States. The EU case definition [2] is shown (Box).

Individual cases of travel-associated Legionnaires' disease are in most circumstances diagnosed and reported by the case's country of residence to the European Surveillance System (TESSy) at ECDC. Case reports include age, sex, date of onset of disease, method of diagnosis and travel information for the different places where the case had stayed within two to ten days before onset of disease. After receiving the report, the TESSy database is searched to determine whether a new case should be classified as a single case or as part of a cluster, according to the following definitions used by the network.

A single case: a person who stayed at a public accommodation site in the two to ten days before onset of illness and the site has not been associated with any other case of Legionnaires' disease in the previous two years.

- A cluster: two or more cases who stayed at the same public accommodation site in the two to ten days before onset of illness and whose onsets were within the same two-year period.

- If there are three or cases or more with onset of disease within the same three-month period, this is called a rapidly evolving cluster and a notification is sent to all tour operators.

If there is a single case, a notification is sent to the country where the accommodation site is situated, with a copy to the reporting country. If the case is a part of a cluster, a notification is sent to all network members. When the accommodation site is outside EU and when a specific contact person for ELDSNet is known, the country concerned is included as a recipient of the notification. All notifications, except those relating to 
domestic travellers, are also sent to the World Health Organization.

When a cluster is detected, a full investigation is required at the accommodation site and preliminary results from the risk assessment and start of control measures should be reported back to ECDC within two weeks of the alert, using the standard operating procedures Form A [5]. The investigation is carried out by regional or local public health authority, depending on the national rules in each country. The investigation and risk assessment carried out are described in the EWGLI technical guidelines for the investigation, control and prevention of travel associated Legionnaires' disease [4].

A second form, Form $B$, is then used to report the results of environmental sampling and the control measures applied at the site back to the coordinating centre in ECDC within a further four weeks, thus allowing six weeks in total for all investigations to be completed. If the forms are not returned within the time frames, or they report that actions and control measures are unsatisfactory, ELDSNet publishes the details of the site associated with the cluster on its website and tour operators are informed about the accommodation site being published. Information is removed from the website when the investigations and control measures are reported to have been satisfactorily completed. If a cluster is associated with more than one accommodation site, it is noted as a 'complex cluster' and all sites stayed at by the cluster cases are subject to the same investigation procedures as described above.

The data presented here is also included in the annual surveillance report for Legionnaires' disease 2010 [5]. In 2010, there were no data from Germany, but they are now part of the travel-associated Legionnaires' disease reporting scheme.

We use in this article a similar reporting format as used in previous publications on travel-associated Legionnaires' disease data, to facilitate comparison [6-9].

\section{Results}

A total of 864 cases of travel-associated Legionnaires' disease with onset of disease in 2010 were reported to EWGLINET/ELDSNet. This is an increase (+5.6\%) compared with the 818 cases reported in 2009 [10], but does not reach the peak of 947 cases observed in 2007 (Figure 1).

Cases were reported from 19 EU/EEA countries (United Kingdom (UK) counted as one country) and two countries outside the EU (United States, 11 cases, and Croatia, 2 cases), as the cases were associated with accommodation sites in the EU. The countries that reported the most cases were France $(n=191)$, the UK $(n=154)$, the Netherlands $(n=148)$ and Italy $(n=142)$ (Table).
TABLE

Cases of travel-associated cases of Legionnaires' disease by reporting country, 2009-10

\begin{tabular}{|c|c|c|}
\hline \multirow{2}{*}{ Reporting country } & \multicolumn{2}{|c|}{ Number of reported cases } \\
\hline & 2009 & 2010 \\
\hline France & 163 & 191 \\
\hline United Kingdom & 173 & 154 \\
\hline Netherlands & 109 & 148 \\
\hline Italy & 169 & 142 \\
\hline Spain & 65 & 67 \\
\hline Denmark & 34 & 32 \\
\hline Norway & 21 & 25 \\
\hline Sweden & 22 & 20 \\
\hline Austria & 17 & 19 \\
\hline Belgium & 12 & 16 \\
\hline United States & 11 & 11 \\
\hline Finland & 6 & 8 \\
\hline Ireland & 2 & 7 \\
\hline Czech Republic & 5 & 5 \\
\hline Malta & 0 & 5 \\
\hline Luxembourg & 2 & 3 \\
\hline Portugal & 4 & 3 \\
\hline Hungary & 2 & 2 \\
\hline Croatia & 1 & 2 \\
\hline Latvia & 0 & 1 \\
\hline Slovenia & 2 & 1 \\
\hline Bulgaria & 1 & 0 \\
\hline Others & 0 & 2 \\
\hline Total & 821 & 864 \\
\hline
\end{tabular}

Source of 2009 data: The European Surveillance System (TESSy) data downloaded 5 August 2011

Among the reported cases, 624 (72.2\%) were male and $240(27.7 \%)$ were female, resulting in a male to female ratio of $2.6: 1$, which was almost identical to the ratio for 2009 (2.7:1) [10].

Cases were reported in all age groups except the youngest one, the median age being 61 years (range: 21-96) in male cases and 63 years (range: 12-95) in female cases. The highest proportion of cases was in the 60-69-year age group (male cases: $n=183$; female cases: $n=82$ ).

Outcome of illness was reported for 514 (59.5\%) cases (voluntary reporting and different definitions are used in the reporting countries). Of these cases, 24 ( $4.7 \%)$ 
Accommodation sites per destination country associated with cases of travel-associated Legionnaires' disease, EU Member States and neighbouring countries, 2010

Number of sites

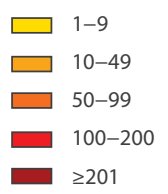

Non-visible countries
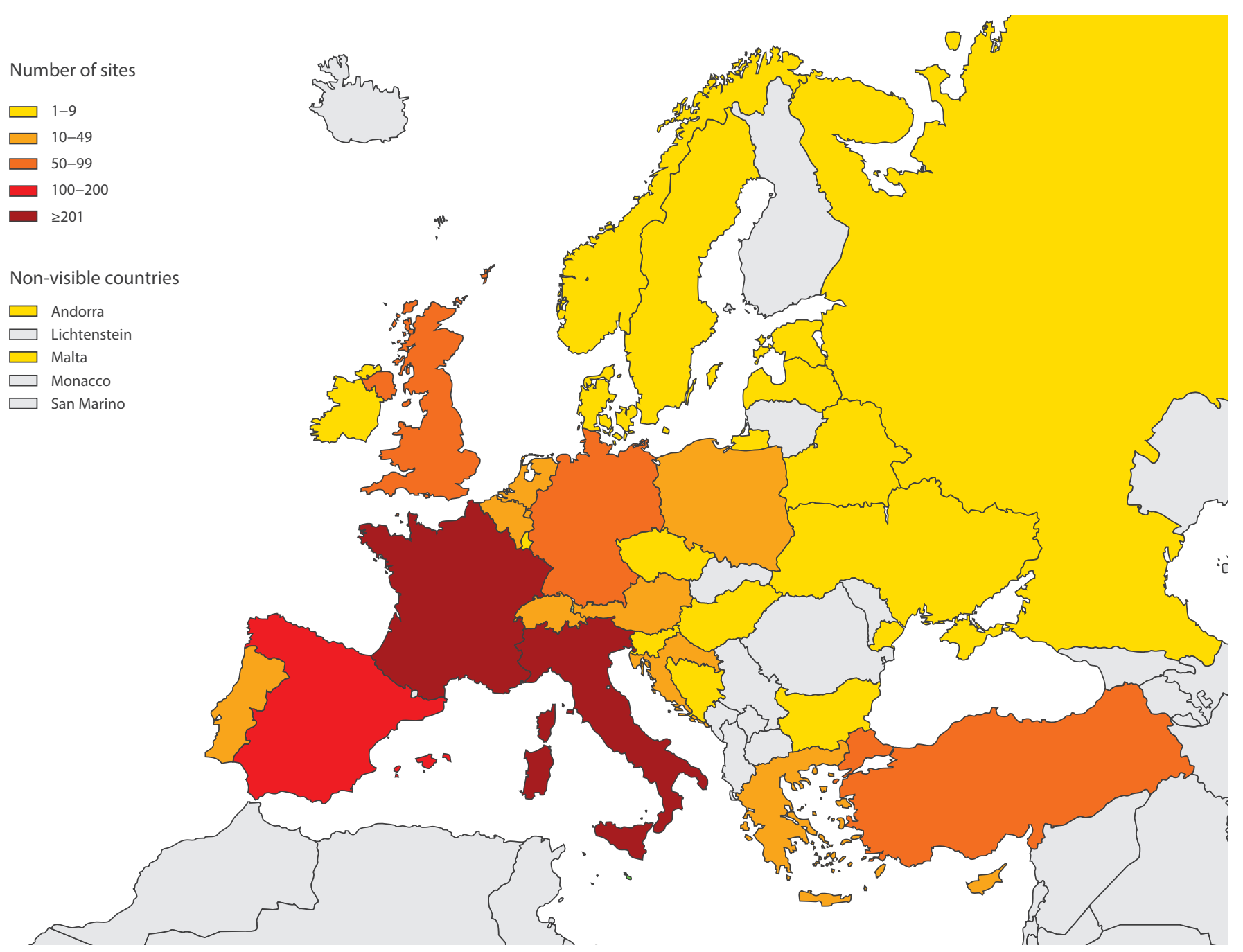

EU: European Union.

Source: [5].

were reported to have had a fatal outcome, almost the same proportion reported in 2009. Of the four female cases who had a fatal outcome, one was aged 58 years and the other three were aged 82 years. The fatal cases among the 20 male cases were aged from 38 years up to 90 years-old; the majority of male cases with a fatal outcome were in the age group 60-69 years.

There is seasonal variation in the onset of travel-associated Legionnaires' disease: with more cases appearing during late summer [6-9]. In 2010, the number of cases peaked in August, with 156 cases, followed by September, with 136 cases. January, February, March, April and December were the months when the lowest number of cases, approximately 30 per month, had onset of disease.

\section{Microbiological analysis}

A total of 809 (94\%) cases in 2010 were reported as confirmed, according to the EU case definition. Of these, 45 (6\%) were diagnosed by culture of the causative organism, a decrease from $10 \%$ in 2009. Of the culture-confirmed cases, 27 were also diagnosed by urinary antigen detection. The vast majority of confirmed cases $(n=762,94 \%)$ were diagnosed by detection of urinary antigen alone. A total of 10 cases (1\%) were confirmed as being due to Legionella pneumophila serogroup 1 by specific antibody response.

The remaining $55(6 \%)$ cases were classified as probable following presumptive diagnosis by single high titre $(n=28,3 \%)$, detection of Legionella spp. nucleic acid $(n=19,2 \%)$ and antibody response specific for L. pneumophila non-serogroup 1 or other Legionella 
Accommodation sites per destination country associated with cases of travel-associated Legionnaires' disease worldwide, 2010

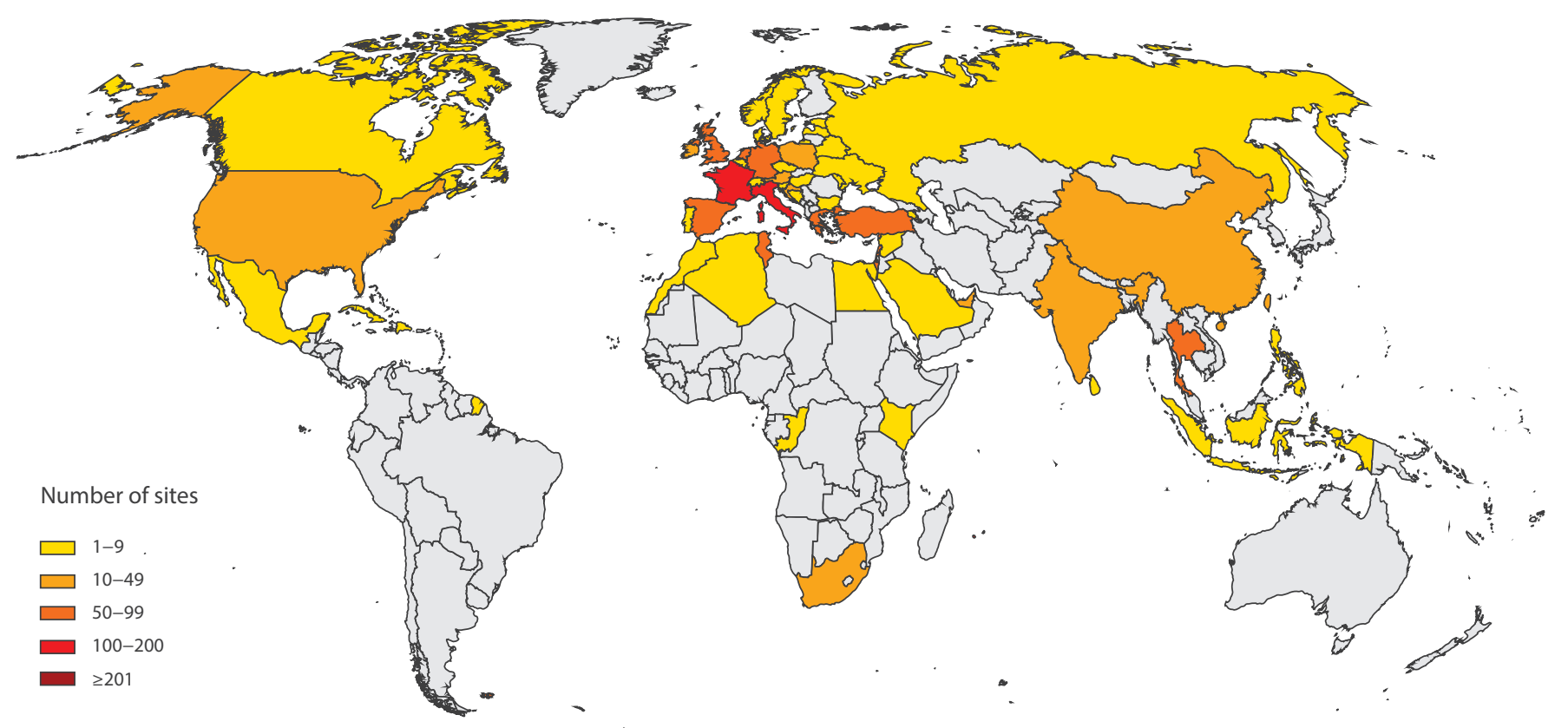

Source: [5].

spp. ( $n=8,1.0 \%)$. Altogether, $672(78 \%)$ cases were reported as being infected with $L$. pneumophila serogroup 1, three with L. pneumophila serogroup 3, two with L. pneumophila serogroup 6, one with L. pneumophila serogroup 12 and three with L. pneumophila mixed serogroups. Furthermore, 158 cases were reported as L. pneumophila serogroup unknown, 1 as Legionella bozemannii and 10 as Legionella species unknown. For 14 cases, the Legionella species was not reported. Sequence-based types were reported for 13 cases (eight from Denmark, four from the UK and one from Austria).

\section{Travel}

The 864 reported cases had made 1,279 visits to accommodation sites around the world.

They visited a total of 66 countries in the 2-10 days before onset of disease. A total of 654 (76\%) cases travelled within the EU: 621 cases visited only one Member State and 33 more than one. Some $20 \%(n=175)$ of cases travelled outside the EU: 166 to a single destination and 9 to more than one non-EU country. A total of 30 cases ( $3 \%$ ) went to both EU and non-EU destinations and 32 cases were associated with cruise ships.

Italy was the country where most cases $(n=209)$ were infected, followed by Spain (177 cases), France (172 cases) and Turkey (48 cases). A total of 169 cases were French residents: $105(62 \%)$ of them visited accommodation sites in France. Likewise, of the 119 Italian residents reported with Legionnaires' disease, 105 (88\%) had visited accommodation sites in Italy.

The number of accommodation sites per destination country with cases of travel-associated Legionnaires' disease is shown in Figures 2 and 3.

\section{Clusters}

A total of 100 new clusters (74 in EU Member States and 26 outside the EU) were detected in 2010, involving 213 associated cases. The largest cluster was associated with a cruise ship and involved 14 associated cases. Italy had the highest number of clusters $(n=24)$ followed by Spain $(n=14)$, France $(n=12)$ and Turkey $(n=10)$. Altogether, clusters in the EU occurred in 13 Member States and on two cruise ships. Outside the EU, 26 clusters occurred in 16 countries and on one cruise ship.

Of the 100 clusters, 44 comprised single cases reported from two or more countries and would probably not have been detected without the European surveillance network. More than $50 \%$ of the clusters $(n=51)$ were detected between July and September.

Complex clusters were more associated with accommodation sites in countries where organised tours to 
Clusters of cases of travel-associated Legionnaires' disease per destination country in EU Member States and neighbouring countries, 2010

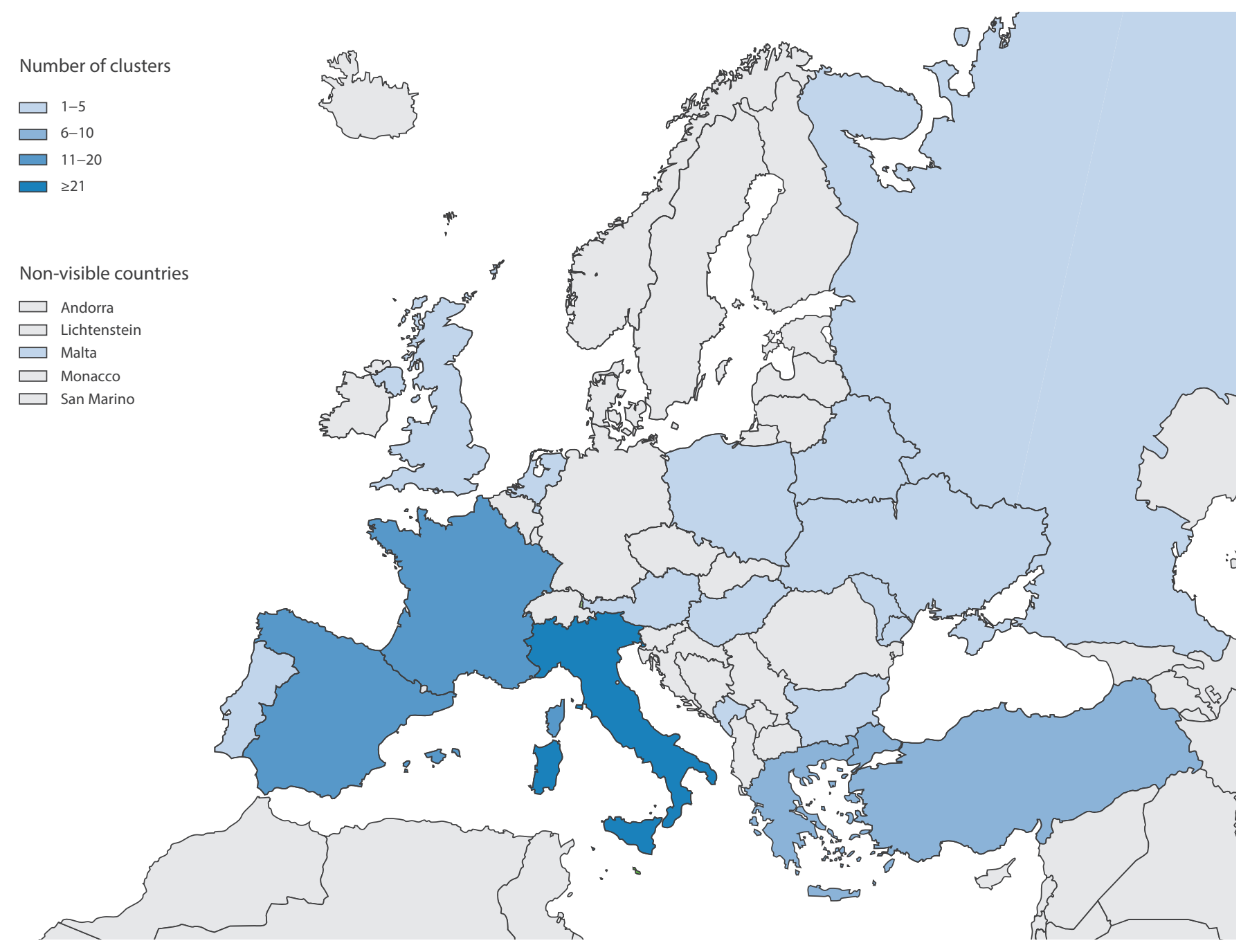

EU: European Union.

Source: [5].

several tourist sites took place, such as China, India, South Africa and Thailand.

Six rapidly evolving clusters were detected: Greece $(n=2)$, Italy $(n=2)$, Spain $(n=1)$ and on a cruise ship $(n=1)$.

The number of clusters per destination country is shown in Figures 4 and 5.

\section{Investigations and publication}

All accommodation sites associated with a cluster of travel-associated Legionnaires' disease ,situated within an EU Member State, should be investigated as described above. In 2010, 100 form Bs were returned to EWGLINET/ELDSNet, reporting detection of Legionella in 61 accommodation sites. The forms were returned not only by Member States but also by several non-EU countries, on a voluntary basis. However, for five sites, form B was not received or the form stated uncertainty regarding the control measures taken, so the names and locations of these sites were published on the ECDC website.

\section{Discussion}

During 2006 to 2008 , the number of cases of travelassociated Legionnaires' disease reported per year had varied from 866 to 947 [7-9]. Legionnaires' disease is still underascertained in most European countries since specific testing for Legionella in patients with pneumonia is not a routine procedure [10]. Furthermore, use of urinary antigen detection as the only laboratory method will lead to underdetection of cases with Legionella non-pneumophila and non-serogroup 1, since the method is designed to detect only L. pneumophila serogroup 1 . It is estimated that only 


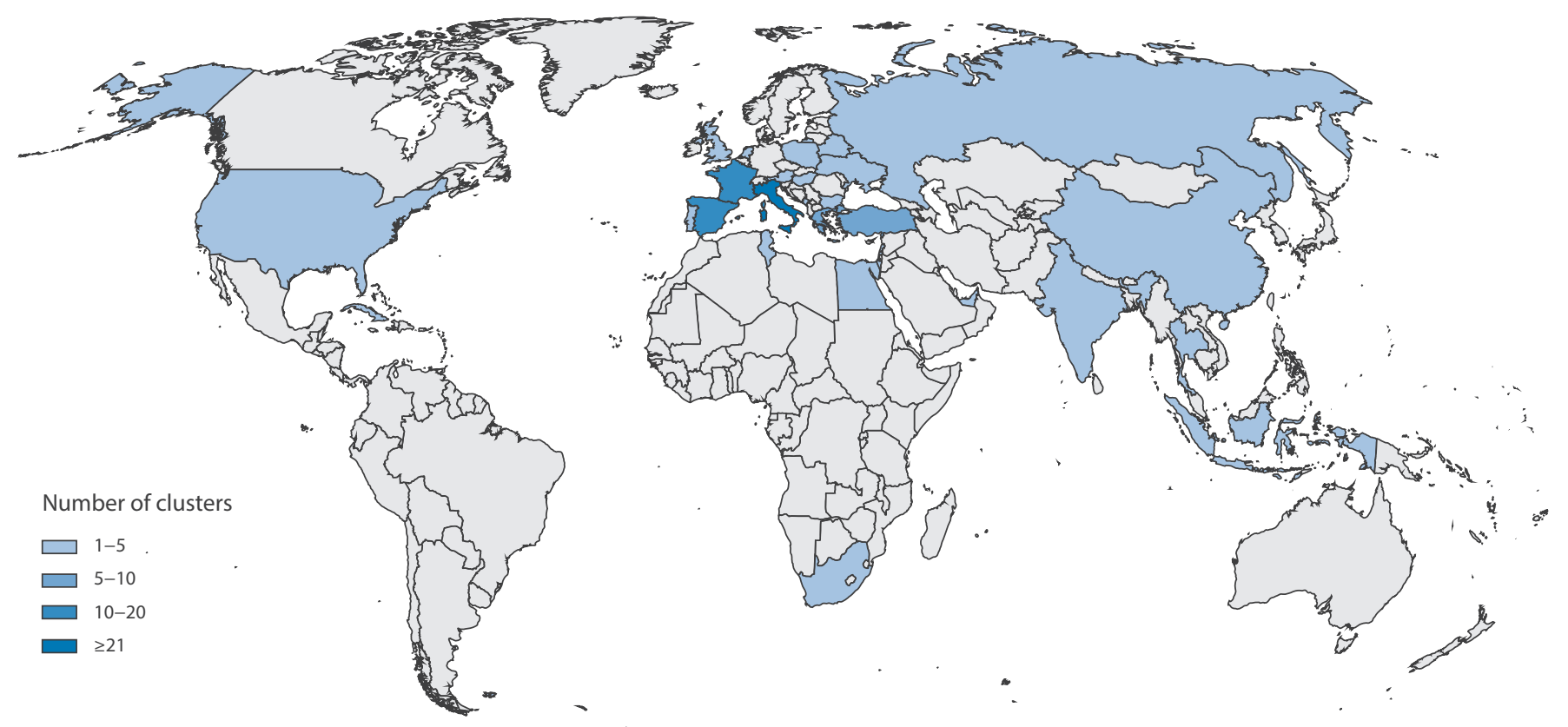

Source: [5].

$10 \%$ of all cases of Legionnaires' disease are notified to public health authorities [10].

For several years, the four countries reporting the vast majority of travel-associated cases have been France, the UK, Italy and the Netherlands. This indicates high awareness of Legionnaires' disease among clinicians in these countries. France and Italy also reported the highest numbers of cases in domestic travellers.

The proportion of cases diagnosed by culture decreased from $10 \%$ in 2009 to $5.6 \%$ in 2010 . However, the decrease was not so drastic when compared with the number of culture-confirmed cases from 2005 to 2008 , when the proportion varied from $4.9 \%$ to $8.2 \%$ [6-9)]. Nevertheless, clinicians should be encouraged to collect more specimens for culturing. It is important to be able to compare clinical isolates with environmental isolates from different sampling sites, to identify the source of infection and prevent any subsequent cases.

The case fatality rate for 2010 (4.7\%) was lower than the $5.8-9.8 \%$ between 2006 and 2009. However, in more than $40 \%$ of the cases, the clinical outcome of the patient was unknown at the time of reporting. In the interest of timely reporting and implementation of control measures as soon as possible at the associated accommodation sites, this incompleteness of outcome data seems acceptable.

The added value of ELDSNet is easier to quantify than for other similar European surveillance networks, in that $44 \%$ of the clusters reported would most probably not have been detected without ELDSNet. Some countries do take action when a single case is reported to be associated with an accommodation site in that country, but in most countries, action is only taken after a cluster notification. Therefore, ELDSNet cluster notifications help to identify accommodation sites that might pose a risk to human health while the control measures implemented prevent further cases of Legionnaires' disease. This is demonstrated by the fact that in 61 of the 100 accommodation sites reported to have been sampled, Legionella bacteria were identified in the water systems.

Despite the challenges and changes in reporting systems with transition of the network to a new coordination centre in April 2010, network members have continued to report cases in a timely manner and undertake cluster management in response to notifications. This highlights the dedication and considerable added value of this network for public health in Europe. 


\section{Acknowledgements}

We would like to thank all network members for reporting and all persons involved in public health programmes for the control and prevention of travel -associated Legionnaires' disease. A list of current collaborating centres can be found on the ECDC website [11]. We would also like to thank Virginia Estevez, ECDC, for her support in creating maps.

\section{References}

1. European Centre for Disease Prevention and Control (ECDC) Legionellosis. Stockholm: ECDC. [Accessed 25 Oct 2011]. Available from: http://ecdc.europa.eu/EN/HEALTHTOPICS/ LEGIONNAIRES DISEASE/Pages/index.aspx

2. European Commission. Commission Decision of 28 April 2008 amending Decision 2002/253/EC laying down case definitions for reporting communicable diseases to the Community network under Decision No 2119/98/EC of the European Parliament and of the Council. Official Journal of the European Union. Luxembourg: Publications Office of the European Union. 18.6.2008:L 159. Available from: http://eur-lex.europa.eu/ LexUriServ/LexUriServ.do?uri=0J:L:2008:159:0046:0090:EN: PDF

3. European Centre for Disease Prevention and Control (ECDC). European Legionnaires' Disease Surveillance Network (ELDSNet): operating procedures. Stockholm: ECDC; 2012 Available from: http://ecdc.europa.eu/en/publications/ Publications/1202-TED-ELDSNet-operating-procedures.pdf

4. EWGLI technical guidelines for the investigation, control and prevention of travel associated Legionnaires' disease. Stockholm: European Centre for Disease Prevention and Control: September 2011. Version 1.1. Available from: http://ecdc.europa.eu/en/activities/surveillance/ELDSNet/ Documents/EWGLI-Technical-Guidelines.pdf

5. European Centre for Disease Prevention and Control (ECDC). Legionnaires' disease in Europe, 2010. Stockholm: ECDC; 2012. Available from: http://ecdc.europa.eu/en/publications/ Publications/SUR-Legionnaires-disease-surveillance-2010.pdf

6. Joseph CA, Ricketts KD, Yadav R, Patel S, on behalf of the European Working Group for Legionella Infections. Travelassociated Legionnaires' disease in Europe in 2009. Euro Surveill. 2010;15(41):pii=19683. Available from: http://www. eurosurveillance.org/ViewArticle.aspx?Articleld=19683

7. Ricketts K, Joseph CA, Yadav R, on behalf of the European Working Group for Legionella Infections. Travel-associated Legionnaires' disease in Europe in 2008. Euro Surveill. 2010;15(21):pii=19578. Available from: http://www. eurosurveillance.org/ViewArticle.aspx?Articleld=19578

8. Joseph CA, Yadav R, Ricketts KD, on behalf of the European Working Group for Legionella Infections. Travel-associated Legionnaires' disease in Europe in 2007. Euro Surveill. 2009;14(18):pii=19196. Available from: http://www. eurosurveillance.org/ViewArticle.aspx?Articleld=19196. PMid:19422777.

9. Ricketts KD, Yadav R, Joseph CA. Travel-associated Legionnaires' disease in Europe: 2006. Euro Surveill. 2008;13(29): pii=18930. Available from: http://www. eurosurveillance.org/ViewArticle.aspx?Articleld $=18930$

10. European Centre for Disease Prevention and Control (ECDC). Legionnaires' disease in Europe 2009. Stockholm: ECDC; 2011. Available from: http://ecdc.europa.eu/en/publications/ Publications/1109_SR_Legionnaires'\%2odisease_ Europe_2009.pdf

11. European Centre for Disease Prevention and Control (ECDC). ELDSNet. Participating institutions. Members of network. Stockholm: ECDC. [Accessed 25 Oct 2011]. Available from: http://www.ecdc.europa.eu/en/activities/surveillance/ ELDSNet/Pages/Participating institutions.aspx 